PERSPECTIVES ON PSYCHOTHERAPY

\title{
Polythematic Delusions and Logico- THEORETICAL VS. EXPERIMENTALIST TURN OF MIND
}

\author{
Paul Franceschi* \\ University of Corsica, Corte, France
}

\begin{abstract}
This article aims to contribute to cognitive therapy of polythematic delusions by proposing a preliminary step to the implementation of traditional cognitive therapy, based on the construction of alternative hypotheses to delusions and testing of the latter. This additional step resides in the construction in the patient of the necessary skills to use the general experimentalist method of knowledge acquisition. Such an approach is based on the contrast between the logico-theoretical and the experimentalist turn of mind. Some elements such as to allow any such construction in the patient are then described and analyzed.
\end{abstract}

Key w ord s: Cognitive therapy; Schizophrenia; Delusions; Polythematic delusions; Metacognition; Logic

\section{IN TRODUCTION}

Classical cognitive therapy targeted at polythematic delusions associated with schizophrenia is based on the search for evidence related to delusional ideas and the construction of alternative hypotheses to the latter. This article aims to contribute to cognitive therapy for polythematic delusions by proposing a preliminary step to this classical cognitive therapy. Such a step aims to strengthen the patient's ability to use the general approach of experimentalist type for knowledge acquisition-an approach which is based on the opposition between the theoretical-logical and the experimentalist turn of mind. Some elements such as to enable the reinforcement of such a capability in the patient are thus described and analyzed.

\section{THEORETICAL-LOGICAL VS. EXPERIMENTALIST TURN OF MIND}

Cognitive therapy of schizophrenia aimed at polythematic delusions includes a component mainly oriented tow ard teaching the patient skills for search of evidence relating to the ideas associated with polythematic delusions, as well as the construction of alternative hypotheses (Kingd on \& Turkington, 1994, 2002). It consists thus, on the one hand, of teaching the patient how to construct alternative hypotheses to delusions such as "television is talking about me", "a satellite sends me thoughts", "aliens are plotting against me", etc., and secondly to encourage him/ her to test both delusions and the associated alternative hypotheses, in order to validate or invalidate them. Such an approach applies then, in a specific way, to polythematic delusions which are inherent to the patient, and the corresponding alternative

*Correspondence to: Paul Franceschi, email: p.franceschi@univ-corse.fr

Received November 15, 2012; accepted March 12, 2013; Act Nerv Super (Praha) 55(1-2), 67-73. 
hypotheses. At this point, we can observe that this approach is associated with a general methodology of the same nature, which is not based on the very polythematic delusions inherent to the patient. Such an approach proves then grounded on the acquisition of the general ability to build up alternative hypotheses and to carry out tests on different hypotheses. Unlike traditional therapy that bears specifically on the content of the polythematic delusions, such an approach presents a general nature and proves likely to refer to any type of hypotheses. We can describe the general nature of such an approach as experimentalist.

We can observe here that the fact that classical cognitive therapy is based on strengthening in the patient the ability to develop alternative hypotheses to delusions and to perform tests on them, implicitly relies on the fact that such an ability is low or deficient in the patient, at least with respect to the delusions developed by the latter. We suggest then to make the wider assumption that the overall ability to develop altern ative hypotheses and tests in order to validate or invalidate ideas, could be low or deficient in the patient, and that the problem encountered with delusions represents the visible part of a more general problem that is inherent to the patient. We also propose that the two above-mentioned elements (test implementation and construction of alternative hypotheses) are also part of an overall ability that also includes additional elements, and can be defined as the ability to implement the experimentalist method of know ledge acquisition.

For the purpose of the present study, it is necessary to further clarify the very notion of general methodology of experimentalist nature. To this end, it is worth contrasting first the experimentalist turn of mind with the logico-theoretical one. Such opposition allows for a better understanding of the experimentalist approach itself. This opposition corresponds essentially to a classical opposition in science, which contrasts two particular styles that each lead to acquisition of knowledge. Whereas the method of experimentalist inspiration proceeds by elaborating hypotheses and testing them, the logico-theoretical method proceeds by logical deduction or induction from a set of knowledge of which the one who exerts it strives to maintain consistency. Both methods, on the scientific level, each have their supporters and detractors. However, advances in knowledge are to be credited to both methods, which ultimately appear as complementary and may eventually be regarded as two ways of accessing scientific knowledge.

The opposition between experimentalist vs. logico-theoretical turn of mind is not limited, however, to the scientific field. Indeed, such opposition has a more general scope and also applies to any body of knowledge, including that resulting from the process of acquiring information and knowledge concerning everyday life. In this context, the logico-theoretical turn of mind notably proceeds by deduction, by trying to acquire knowledge in a logical way; it is aimed at explaining and interpreting facts and phenomena. Such an approach is underpinned by a concern for consistency of the whole corresponding set of knowledge, by also trying to identify and remedy any internal contradiction. The logico-theoretical approach may also proceed by inductive reasoning, thus making use of inductive generalization. In addition, when an internal contradiction is found, thus rendering the whole set of knowledge inconsistent, the one who proceeds in a logico-theoretical way strives to quickly restore this consistency, by possibly modifying some elements that are part of the overall knowledge at his/ her disposal. Conversely, the one who proceeds with the help of the experimentalist approach is basically concerned with validating theories and hypotheses, through experimentation, testing, and search for evidence. He/ she is then concerned with only retaining ultimately that knowledge that has been validated by experience and whose strength lies in the evidence which has been thus collected.

Thus characterized through their opposition, it is no less apparent that each of the experimentalist or logico-theoretical method of knowledge acquisition has its drawbacks when pushed to the extreme. When applied in excess, the logico-theoretical method thus leads to risky speculation and lack of evidence, to unrealistic and disconnected from the reality viewpoints. Conversely, experimentation pushed to the extreme leads to knowledge that lacks power of abstraction, explanatory and predictive power, and does not allow 
understanding of the data and the phenomena. In order to better emphasize the related notions and also better highlight the relationship with the different neighboring concepts, it is worth providing some additional insight over the opposition between experimentalist and logico-theoretical methodology. We shall propose then to make use of the matrices of concepts, which allow to emphasize the relationship s between some given concepts. A matrix of concepts thus consists of six concepts, distributed along two dual poles: A and A. Each of these poles admits of respectively a concept of neutral $\mathrm{A}^{0}, \overline{\mathrm{A}}^{0}$, positive $\left(\mathrm{A}^{+}, \overline{\mathrm{A}}^{+}\right)$and negative $\left(A^{-}, \bar{A}^{-}\right)$nature. In total, the matrix consists of the six following concepts: $A^{+}, A^{0}, A^{-}, \bar{A}^{+}, \bar{A}^{0}, \bar{A}$.

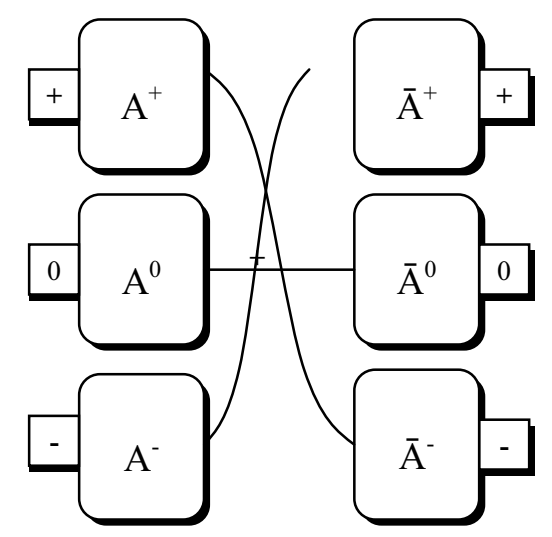

Figure 1. Structure of a matrix of concepts

Among the main relationships that can be defined between concepts of the same matrix, it is worth citing: (i) the duality relation, between two neutral concepts of different poles: $\mathrm{A}^{0}$ and $\overline{\mathrm{A}}^{0}$; (ii) the relation of antinomy (or of contrary) between two concepts that are not neutral (that is to say, positive or negative) of opposite polarity and of different poles: $\mathrm{A}^{+}$and $\overline{\mathrm{A}}^{-}$, as well as $\mathrm{A}^{-}$and $\overline{\mathrm{A}}^{+}$; (iii) the complementary relationship between two concepts of same polarity (positive or negative) and of different poles: $\mathrm{A}^{+}$and $\overline{\mathrm{A}}^{+}$as well as $\mathrm{A}^{-}$and $\overline{\mathrm{A}}^{-}$. For example, the matrix of concepts corresponding to the concepts of courage, cowardice and temerity is thus as follows:

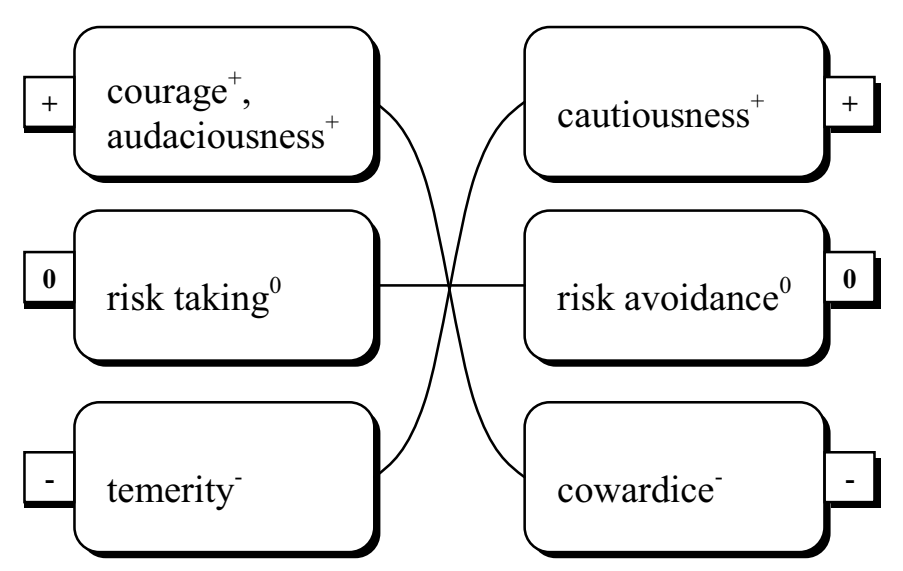

Figure 2. An instance of a matrix of concepts

At this step, we are in a position to construct the matrix of concepts that applies to the opposition between theoretical-logical and experimentalist turn of mind. Each of these concepts presents an intrinsic neutral nature, but is likely to give rise to a positive and a negative form. The corresponding matrix of concepts is thus the following: 


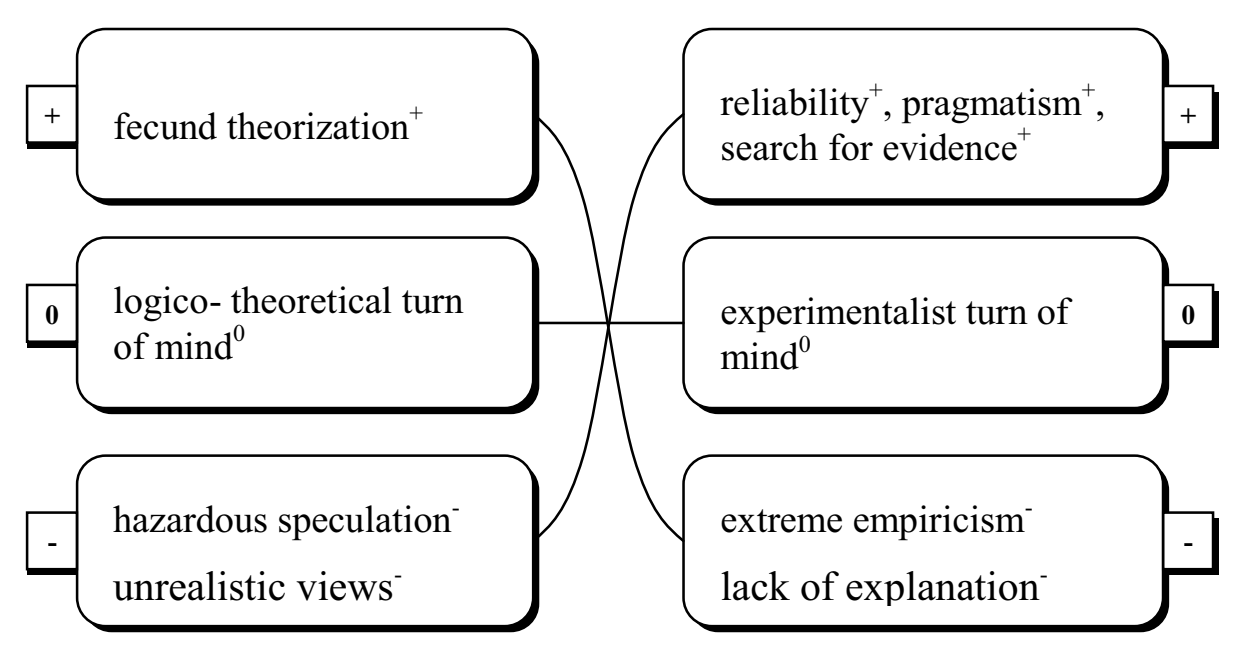

Figure 3. The matrix of concepts associated with the theoretical-logical/ experimentalist turn of mind

We can see here that the theoretical-logical turn of mind presents a positive form which leads to fruitful theorization, and a negative form that engenders unrealistic ideas and hazardous speculations. Conversely, the experimentalist turn of mind has a positive form which entails the solidity of knowledge and the search for evidence, and a negative form which leads to extreme empiricism, associated with lack of understanding and absence of explanation.

\section{GENERAL APPLICATION OF THE EXPERIMENTALIST METHOD OLOGY}

The foregoing analysis leads to the hypothesis that it might be advisable to rebuild preliminarily in the patient the general ability to implement the experimentalist method of knowledge acquisition, before applying it later in a specific way to polythematic delusions. Cognitive therapy targeted at polythematic delusions would accordingly involve two phases: the first aimed at restoring in the patient the functional ability to the experimentalist general approach; and the second intended to implement the latter, in a specific way, by applying it to polythematic delusions. Several arguments seem to lean in favor of such an option. Firstly, it seems preferable that the patient be first convinced of the merits of the experimentalist method of acquiring knowledge, even before applying it to polythematic delusions. In other words, it seems better than the patient has himself/ herself acquired first the belief that the theoretical-experimental method is effective and useful before applying it to the specific topics corresponding to delusions. Second, it also seems preferable that the patient would acquire a prior good practice and be exercised first to the experimentalist method on external, neutral and impersonal facts, before applying it to his/ her own delusions which present for him/ her an eminently emotional, personal and sensitive nature. Presumably also the choice of topics external to the patient should be such as to enable him/ her to mobilize his/ her cognitive abilities optimally. Thirdly, it is reasonable to think that the fact of applying the experimentalist methodology exclusively to the content of polythematic delusions, without possessing at the same time such a general ability might have some disadvantages. One of these drawbacks may lie in the fact that the patient could quickly rebuild some other delusions from other themes than those usual to him/ her. Finally, it is worth mentioning that the acquisition and the general practice of the experimentalist methodology on topics that are 
completely independent of the content itself of the polythematic delusions should be nonconfrontational, and likely to preserve the therapeutic alliance.

At this point, it is possible to sketch an outline of what could consist such a preliminary part of cognitive therapy for polythematic delusions. This last part would include a component targeted at learning the construction of alternative hypotheses, and a component designed to the learning of testing different hypotheses. However, in order to form a coherent whole, it is reasonable to think that this part of the therapy should also include an explanation of a number of related concepts, among which we can mention: the distinction between fact and hypothesis; the notion of proof; the distinction between evidence and conviction; the notion of validation and invalidation of a hypothesis; the distinction between fact and interpretation of fact; the distinction between fact and perception of fact; the distinction between fact and fact narration; the construction of alternative hypotheses; the development of tests with regard to a hypothesis; the causal relationship between facts; the proof of the causal relationship; the distinction between facts whose cause is intentional or non-intentional; the notion of explanation of a given phenomenon. Several of these elem ents, especially those related to the construction of alternative hypotheses, are integral part of the training program for metacognition developed by Moritz et al. $(2010,2011)$.

It is also useful to point out here several areas where the above-mentioned principles could be put into practice. These areas are potentially very diverse, but it is however possible to describe more accurately some of them, which relate to electronics and computer science. In the field of electronics first, consideration could be given to take an interest in electronic circuits (or computer simulations thereof) and their operation. Thus, the patient's attention could focus on the operational problems of such and such circuit and especially on the search for causes of observed failures (e.g. the fact that a led indicator does not light up). It will then be necessary to formulate different hypotheses regarding the cause of the malfunction, which may relate to different circuit components (transistors, power supply, the led indicator itself, etc..) which can then be tested in order to be validated or invalidated, and later give rise, depending on the test results to other hypotheses, etc..

The field of computer science, second, could offer various fields of applications, especially in programming. We may notably consider a computer program that is supposed to produce a given result but has a defect in its execution, due to a "bug". It will be then a matter of accurately determining the cause of this bug i.e., of finding the specific instruction within the program, which is responsible. The patient would thus be required to make assumptions regarding the specific instruction in the program among instr ${ }_{1}$, instr $_{2}$, inst $_{3}, \ldots$, instr $r_{\mathrm{n}}$, which is at the origin of the bug and to test successively the latter. This will lead for example to eliminate the instruction instr $r_{1}$ of which it will be assumed that it is the origin of the bug and to test the program without it, etc.. If the latter test invalidates the hypothesis, such an approach will then lead to test another instruction $i n s t r_{2}$, and so on.

\section{SPECIFIC APPLICATION OF THE EXPERIMENTALIST METHODOLOGY TO POLYTHEMATIC DELUSIONS}

Classical cognitive therapy of schizophrenia (Kingdon \& Turkington, 1994; Beck \& Rector, 2000; Kingdon \& Turkington, 2002) aims to gradually reduce the degree of belief in the patient's delusions. For this purpose, the therapist suggests to the patient, in a spirit of dialogue of Socratic inspiration to build alternative hypotheses. $\mathrm{He} /$ she also teaches to the patient the process of testing the various competing hypotheses by seeking evidence, thus allowing to confirm or refute them.

It seems useful, at this point, to describe the different stages that occur differently depending on the level—primary, secondary or tertiary—of the corresponding delusions. We shall consider in turn each of these levels. We propose to analyze here the delusions of reference, given that the analysis can be transposed to the delusions of influence, of telepathy, of thought projection or of control. 
A primary delusional argument of reference, first, has the following structure (the symbol $\therefore$ denotes the conclusion):

(R1) in $T_{1}$ I was drinking an aperitif

$\operatorname{premiss}_{1}$

(R2) in $T_{2}$ the presenter of the show said: "Don't drink!"

premiss $_{2}$

(R3) $\therefore$ in $\mathrm{T}_{2}$ the presenter of the show said: "Don't drink!" because in $\mathrm{T}_{1} \mathrm{I}$ was

conclusion drinking an aperitif

The corresponding delusional idea is that according to which the presenter said in $\mathrm{T}_{2}$ : "Don't drink!" because the patient has been drinking an aperitif in $\mathrm{T}_{1}$. The structure of such a delusional idea is as follows: the event $E_{1}$ (in $T_{1} I$ was drinking an aperitif) is the cause of the event $E_{2}$ (in $T_{2}$ the presenter of the show said: "Don't drink!"). In this case, the logical structure of the alternative hypothesis to the delusional conclusion (R3) is that the event $E_{1}$ which is internal to the patient is not the cause of the external event $\mathrm{E}_{2}$. The different alternative hypotheses identify then themselves with alternative causes to the event $\mathrm{E}_{2}$. Thus, the delusional conclusion (R3) may be confronted with an alternative hypothesis such as: the presenter said in $\mathrm{T}_{2}$ : “Don't drink!" because the script of this television program contained it. Another alternative hypothesis is that it is the assistant presenter who suggested to say it, etc..

One may think, however, that the fact of proposing to the patient alternative hy potheses to the delusional conclusion (R3) just mentioned, could prove insufficient. In effect, the patient's delusional idea that the event $\mathrm{E}_{1}$ internal to the patient is the cause of the external event $\mathrm{E}_{2}$, not only has the nature of a hypothesis, but also has explanatory power, in the sense that it constitutes an explanation of the fact that appears bewildering to the patient that the presenter has said: "Do not drink!" immediately after the patient has been drinking an aperitif. In comparison, the fact that the event $\mathrm{E}_{2}$ internal to the patient is not the cause of the external event $\mathrm{E}_{2}$, constitutes an alternative hypothesis, but proves devoid of such explanatory power. For this reason, we believe that the mere statement, under this form, of the latter alternative hypothesis should not suffice to gain the support of the patient. For it is necessary to submit to the latter an alternative hypothesis to the conclusion (R3), which is also able to provide an explanation for the rapid succession of events $E_{1}$ and $E_{2}$. In this context, an alternative hypothesis that also allows to provide an explanation for the rapid succession of two phenomena, is the one according to which the external event $E_{2}$ succeeded immediately after internal event $\mathrm{E}_{1}$, by the effect of a coincidence. Under these conditions, the patient faces two competing hypotheses that may explain the rapid and disturbing sequence of events $E_{1}$ and $\mathrm{E}_{2}$ : the first hypothesis being that $\mathrm{E}_{1}$ is the cause of $\mathrm{E}_{2}$; and the second being that the rapid succession of $E_{1}$ and $E_{2}$ is but a coincidence.

Secondly, the structure of secondary delusional arguments of reference is as follows:

$$
\text { in } \mathrm{T}_{2} \text { the presenter of the show spoke according to what I was doing }
$$

premiss $_{1}$

$$
\text { in } \mathrm{T}_{4} \text { the presenter of the show spoke according to what } \mathrm{I} \text { felt }
$$

premiss $_{2}$

in $\mathrm{T}_{6}$ the presenter of the show spoke according to what I was doing

premiss $_{3}$ $\ldots$

(R10) $\quad \therefore$ the presenters of the shows speak according to what I do or what I feel

conclusion

The corresponding delusional idea of reference is then the conclusion (R10) that the presenters of the shows speak according to what the patient makes or feels. The conclusion (R10) is of an inductive nature and constitutes a generalization from the several instances (R1), (R2), (R3), ... Here, the logical structure of the alternative hypothesis to the conclusion (R10) is that the presenters of the shows do not speak according to what the patient makes. But in the same way as above, such a hypothesis proves devoid of explanatory power. In contrast, the alternative hypothesis, which has an additional explanatory power, is the fact that by the effect of coincidences, the rapid succession of two events that may give the impression of the existence of a relationship causality, occurs frequently. 
Finally, the ternary delusional arguments of reference exhibit the following structure:

(R10) $\therefore$ the presenters of the shows speak according to what I do or what I feel

premiss

(R11) $\therefore$ television speaks about me

conclusion

The ternary delusional idea of reference is the one according to which television speaks of the patient. The logical structure of the alternative hypothesis is the one under which television does not speak of the patient. However, in the same way as above, such a hypothesis does not possess in itself an explanatory power. For the conclusion (R11) has, in the patient's mind, an explanatory function to the succession of events that he/ she experienced. It proves thus necessary, at this stage, to propose an alternative explanation, which resides in the fact that through the effect of coincidences, it frequently happens that the patient's internal events are immediately followed by external events, which can give the impression that there is a causal relationship between the two successive events. However, it may be pointed out to the patient, there is a much larger number of pairs of successive events that are not consistent with a causal relationship. It is indeed a common attitude to pay attention only to the succession of two events that could be meaningful, even though it occurs every day many more successions of two unmeaningful events and to which one does not pay any attention. This appears as a special case of misinterpretation of random data (Bressan, 2002).

\section{CONCLUSION}

At this point, it is worth translating the previous elements in terms of testable hypotheses by the clinician. This leads thus to test the hypothesis that cognitive therapy applied to polythematic delusions may be more effective if it included two successive steps: the first advocated by the present study that aims to reconstruct the patient's general ability to acquisition of knowledge through the practice of the experimentalist method; and the second, classically defined by cognitive therapy of schizophrenia that leads to apply specifically the skills thus acquired to the content of polythematic delusions.

Finally, it is possible to synthesize the ideas regarding the co-morbidity of schizophrenia with the elements resulting from the present study. We are thus able to define the different stages of the resulting process for cognitive therapy of delusions inherent to schizophrenia. This would mean thus, in a first step, determining the co-morbid disorders (specific phobias, generalized anxiety disorder, social phobia, intermittent explosive disorder, etc.). associated in the patient with the delusional ideas and to apply first a specific cognitive therapy. In a second step, it would mean applying the learning phase of the above-mentioned method experimentalist of a general nature. Finally, in a final phase, it should be proceeded as indicated by classical cognitive therapy, by applying specifically the experimentalist methodology to the content of delusions. This can be translated as follows in terms of testable hypotheses: a cognitive therapy of schizophrenia that would proceed according to these three successive stages could be more effective than classical cognitive therapy.

\section{REFEREN CES}

Beck, A. (2002). Delusions: A Cognitive perspective. Journal of Cognitive Psychotherapy, 16-4, 455-468.

Bressan, P. (2002). The connection between random sequences, everyday coincidences, and belief in the paranormal. Applied Cognitive Psychology, 16, 17-34.

Kingdon, D. \& Turkington, D. (1994). Cognitive-behavioural Therapy of Schizophrenia. New York: Guilford.

Kingdon, D. \& Turkington, D. (Eds.) (2002). The Case Study Guide to Cognitive Behaviour Therapy of Psychosis. Chichester: Wiley.

Moritz, S., Woodward, T. S., \& Metacognition Study Group. (2010). Metacognitive training for schizophrenia (MCT). Manual (4th ed.), Hamburg: VanHam Campus.

Moritz, S., Kerstan, A., Veckenstedt, R., Randjbar, S., Vitzthum, F., Schmidt, C., Heise, M., Woodward, T.S. (2011). Further evidence for the efficacy of a metacognitive group training in schizophrenia. Behaviour Research and Therapy, 49, 151-157. 